\title{
Statistical analyses of Microwave type III bursts and CMEs
}

\author{
Y. Ma ${ }^{1} \dagger$ D. Y. Wang ${ }^{2}$ and Y. H. Yan ${ }^{3}$ \\ ${ }^{1}$ National Astronomical Observatories/Yunnan Observatory, Chinese Academy of Sciences \\ Kunming, 650011, P.R. China \\ email: mayuanf@yahoo.com.cn \\ ${ }^{2}$ Purple Mountain Observatories, Chinese Academy of Sciences, 210008 Nanjing, P.R. China \\ ${ }^{3}$ National Astronomical Observatories, Chinese Academy of Sciences, 100012 Beijing China
}

\begin{abstract}
The Statistics analyses of the microwave type III bursts, coronal mass ejections (CMEs), H $\alpha$ flares and relevant events observed with $5200-7600 \mathrm{MHz}$ spectrograph at the Chinese National Astronomical Observatory during the 23rd solar cycle are carried out in this article. Some significant results are obtained from the relevant events. The radiation mechanism of that is also discussed from the observation characteristics.
\end{abstract}

Keywords. Microwave type III bursts, $\mathrm{H} \alpha$ flares, CMEs

\section{Data processing}

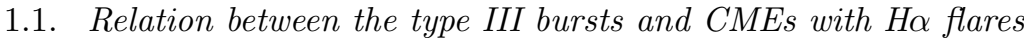

129 type III bursts were recorded with the 5200-7600 MHz spectrograph at the Chinese National Astronomical Observatory during the 23rd solar cycle (from Agust in 1999 to Octerber in 2003). If the comparison is made only from the angle of time, there are 51 type III bursts corresponding to the CMEs, amounting to $40 \%$ of the total bursts, and 43 type III bursts corresponding to the $\mathrm{H} \alpha$ flares. However, since the radio spectrograph has no spatial resolution one can not microwave the active region from which the type III bursts come though the temporal and frequency resolutions of the spectrograph are very high. Therefore, if the relation between the type III bursts and CMEs is considered only from the angle of time, the error would be very great because these type III bursts and CMEs do not come from the same position though they occur simultaneously. For this, the authors only selected the type III bursts which occurred with the CMEs and $\mathrm{H} \alpha$ flares simultaneously for the statistics. The identification and selection of the data are carried out according to the following steps: Firstly gather the type III bursts and the time and regional positions of the CMEs given in the Lasco CME/C2 table, and then gather the data of the $\mathrm{H} \alpha$ flares corresponding to the CMEs, with both with the same time (40 minutes) and the same position ( the CME position is also the two dimensional one ) being regarded as the coexistent events of the type III bursts, CMEs and H $\alpha$ flares, accounting for $33 \%$ of the total type III bursts).

Solar flares are generally divided into two sorts: the pulse flares and gradual flares: 1) The pulse flares: with the duration being less than or equal to 10 minutes. 2) The gradual flares: with the duration being greater than 10 minutes. Among the 129 events recorded during the 23rd solar cycle there were $43 \mathrm{CME}$ events corresponding to the H $\alpha$ flares, 4 pulse flares occurred before the CMEs, with the occurrence being in the first 4 minutes on average. 35 gradual flares occurred before the CMEs, with the occurrence being in the

$\dagger$ Present address: P.O.Box 110, Kunming, 650011, China 
first 29 minutes on average and 4 occurred after the CMEs, with the occurrence being in the last 4 minutes on average.

\subsection{Relation between the type III bursts and CMEs}

The statistics of the events which occurred within 2 hours before the CMEs are collected, with 10 minutes being the time interval, from which it can be seen that most of the microwave type III bursts occurred in 50 to 60 minutes before the CMEs.

\subsection{Initial frequency of the type III bursts and the relation between the frequency drift rate and CMEs}

For understanding the position of the electronic acceleration, the authors gathered the statistics of the initial frequencies of the microwave type III bursts and the CMEs. It is that most of the initial frequencies occur at 5200-5300 MHz. The frequency drift rate is one of the main characteristics, the quickness and slow lines and the positive and negative of the drift rate show the electrons velocity and direction of motion. The authors also gathered the statistics of the relations between the frequencies drift rates of the decimetric type III bursts and CMEs. It is that most of them occur at 1500-2000 $\mathrm{MHz} / \mathrm{s}$, and the frequency drift rates of the microwave type III bursts corresponding to the CMEs principally are those of the fast drift type III bursts.

\section{Discussions}

We think from the above statistics and analysis that:

1. For the pulse flares, we hold that the high-energy electrons of the microwave type III bursts should be accelerated in the process of the flares and they escape outwards along the open magnetic lines of force in the corona, thereby producing the type III bursts. But around the electronic beam along the open magnetic lines the flare ejects plasmoid and the plasmoid forms small scale CMEs, the type III bursts and CMEs are produced in the same flare.

2. For the gradual flares, a prominence may be formed when a magnetic flux tube in the flare floats upwards continuously, the prominence ejects mass to produce large scale CME, but there are open magnetic lines at the sides of magnetic flux tube, the type III bursts are formed by the ejection of energetic electron flux.

3. For the gradual flares that correspond to CMEs, they occur before or after CMEs, the flares that happen before CME are more than those happen after CME. For the flares that happen after $\mathrm{CME}$, it is possible that the propagating distance of the electronic beam from the magnetic reconnection place to the photosphere, is longer than that from the magnetic reconnection place to the corona, therefore, the time when $\mathrm{H} \alpha$ flare occurs is more later than that CME occurs.

\section{Acknowledgements}

This study is supported by NFSC projects of China (grant Nos. 10333030; 10473020; 10425312; 10373026; 40574065).

\section{References}

Y Gopalswamy, \& M Kaiser, 2001, ApJ, 548, 94

Harrison R, 1995, A\&A, 304, 585

L Jun, 2003, New Astronomy Reviewsin 47, 53 